\title{
Osteoporosis management in older patients who experienced a fracture
}

This article was published in the following Dove Press journal:

Clinical Interventions in Aging

22 August 2016

Number of times this article has been viewed

\author{
Mark J Oertel' \\ Leland Graves' \\ Eyad Al-Hihi ${ }^{2}$ \\ Vincent Leonardo ${ }^{3}$ \\ Christina Hopkins ${ }^{2}$ \\ Kristin DeSouza ${ }^{2}$ \\ Rajib K Bhattacharya' \\ 'Division of Endocrinology, \\ Metabolism and Genetics, Department \\ of Medicine, ${ }^{2}$ Department of Internal \\ Medicine, ${ }^{3}$ Department of Enterprise \\ Analytics, University of Kansas \\ Medical Center, Kansas City, KS, USA
}

Correspondence: Rajib K Bhattacharya Division of Endocrinology, Metabolism and Genetics, Department of Medicine, University of Kansas Medical Center, 390I Rainbow Boulevard, Mailstop 2024, Kansas City, KS 66160, USA

Email rbhattacharya@kumc.edu
Background: Fractures in older patients are common, morbid, and associated with increased risk of subsequent fractures. Inpatient and outpatient management and treatment of fractures can be costly. With more emphasis placed on quality care for Medicare beneficiaries, we studied if patients were receiving proper screening for osteoporosis and treatment after diagnosis of fracture. This study aims to determine if adequate screening and treatment for osteoporosis occurs in the postfracture period.

Methods: A retrospective analysis of Medicare beneficiaries aged 67 years or older was gathered from a single institution in both inpatient and outpatient visits. Based on International Classification of Diseases ninth revision codes, primary diagnosis of fractures of neck and trunk, upper limb, and lower limb were obtained in addition to current procedural terminology codes for fracture procedures. We studied patients who had been screened for osteoporosis with a bone mineral study or received osteoporosis treatment after their fracture.

Results: Medicare beneficiaries totaling 1,375 patients were determined to have an inclusion fracture between June 1, 2013 and November 30, 2014. At the time of our analysis on December 1, 2014, 1,219 patients were living and included in the analysis. Of these patients, 256 (21.0\%) either received osteoporosis testing with bone mineral density or received treatment for osteoporosis. On sex breakdown, 208/820 (25.4\%) females received proper evaluation or treatment of osteoporosis in comparison to 48/399 (12.0\%) males. This is in comparison to the Centers for Medicare and Medicaid Services' national average of $19.1 \%$ for osteoporosis management in females.

Conclusion: A minority of studied patients received evaluation or treatment for osteoporosis after their fracture. Postfracture investigation and treatment for osteoporosis in Medicare beneficiaries is inadequate. If improved, Medicare costs could be reduced by prevention of future fractures. Future studies could determine how best to ensure this intervention occurs.

Keywords: osteoporosis, inpatient management, postfracture evaluation, post-fracture treatment

\section{Introduction}

Osteoporosis is a common, debilitating, yet under recognized disease in many older patients. Unfortunately, fractures are the initial clinical presentation. These can carry high mortality risk and lead to decreased functional status. Fractures often require expensive inpatient admissions and surgical corrections. Furthermore, a history of any fragility fracture is a strong risk factor for subsequent fracture. In spite of wide availability of bone mineral density (BMD) testing and availability of several antiosteoporosis medications, a majority of patients following fragility fractures are neither screened for osteoporosis nor treated. ${ }^{1}$

Current recommendations to initiate treatment for osteoporosis are based on history of fragility fracture, BMD that is $<2.5$ standard deviations below the BMD of a young adult reference range, and if Fracture Risk Assessment Tool demonstrates 10-year risk 
of $>3 \%$ for hip fracture or $>20 \%$ for major osteoporotic fracture. With many fragility fractures first identified in the inpatient setting, it has been well established that post-fracture osteoporosis evaluation and treatment was uncommon. ${ }^{2-4}$

By 2025, annual fracture and costs are expected to rise by almost $50 \%$, incurring US $\$ 25.3$ billion in health care costs. ${ }^{5}$ This rise is due to increased incidence in an aging population seeking medical care. Osteoporotic fractures are responsible for almost 2.5 million medical office visits, 432,000 hospital visits, and 180,000 nursing home admissions each year. ${ }^{6}$

As overall life expectancy has increased over the past decades, so has the amount of patients reaching the age of 67 years or older. This has increased the utilization rate and costs of the Medicare program, which is a national health insurance program for legal residents of USA who are $>65$ years old. Improvement in post-fracture evaluation for osteoporosis and treatment rates can both reduce health care costs and patient morbidity and mortality. The aim of this study is to identify and characterize Medicare patients aged 67 years or older who do not undergo osteoporosis treatment or evaluation and ultimately prevent osteoporosis afterward.

\section{Methods}

This was a retrospective, observational analysis of Medicare patients carried out at a single Midwestern university-based academic hospital, which is a large referral center. We studied Medicare patients aged 67 years or older who suffered a fracture within the last 18 months and had received a BMD test or prescription for a drug to treat or prevent osteoporosis afterward. Age 67 years was chosen based on previously published Medicare data provided by Centers for Medicare and Medicaid Services. We chose similar age criteria in our study to compare their results. A 3-month lag period was included to allow a fracture captured late in our encatchment dates to allow appropriate evaluation and treatment for osteoporosis by physicians. Data were obtained from inpatient and outpatient diagnosis codes from the electronic medical record and electronic billing service from February 1, 2013 to July 31, 2014. Due to the fact that this was a quality improvement study, the institutional review board at the University of Kansas deemed ethical approval was not necessary.

The denominator included diagnosis and procedures that identified clinically relevant fractures or procedures to treat fractures. Fractures that were included were obtained from International Classification of Diseases ninth (ICD-9) revision codes, including 733 (osteopathies, chrondropathies, and acquired musculoskeletal deformities but osteoporosis was excluded), 805-809 (fracture of neck and trunk), 810-819 (fracture of upper limb), 820-829 (fracture of lower limb), 79 (reduction of fracture and dislocation), 81.65, and 81.66 (other procedures on spine), and current procedural terminology codes of surgical procedures on the musculoskeletal system. Excluded was 822-825 (fracture of lower leg) to eliminate ankle, forefoot, and toe fractures. Excluded were patients with transportation accident-induced fractures or pathologic fractures.

The numerator included patients who had an inclusion fracture or surgical procedure and received BMDs or osteoporosis medications. Osteoporosis medication included bisphosphonates; calcitonin; hormone therapy, including estrogen, teriparatide, and pamidronate; and the immunological agent denosumab.

\section{Data sources and extraction}

University of Kansas Medical Center clinical data repository, Healthcare Enterprise Repository for Ontological Narration (HERON) was used to query and extract the data for analysis. HERON is an integrated repository of University of Kansas Medical Center patient data, using the open-source i2b2 data architecture and querying platform. Refreshed monthly, HERON joins data from various sources to provide an extended electronic health record across the continuum of care, including Epic (hospital electronic medical records) and IDX (clinical billing system).

After being extracted from HERON, the data were loaded into $\mathrm{R}$ (an open-source statistical analysis programming tool; https://CRAN.R-project.org/), where further cohort identification and characterization was performed. An aggregated patient-level dataset was created to provide for further statistical analysis.

\section{Statistics}

Chi-square analysis was performed for assessing the association of individuals who were screened with a bone density or treated with osteoporotic medication and different comorbidities (sex, age, diabetes, cancer, and chronic kidney disease) (Table 1).

\section{Results}

From February 1, 2013 to July 31, 2014, 6,850 patients were identified as having sustained a fracture after excluding traumatic fractures. A total of 1,375 patients sustained fracture after the age of 67 years. Of these patients who met the inclusion criteria, 1,219 were living at the time of data extraction on December 1, 2014. Deceased patients were excluded from further analysis. Patients with chronic kidney disease stage IV or V were removed from the analysis as many osteoporosis medications are contraindicated in this population. 
Table I Descriptive analysis of post-fracture health care by sex, age, and comorbid conditions

\begin{tabular}{lllll}
\hline Category & \multicolumn{3}{l}{ Individuals who sustained a fracture (N=I,2 I 9) } \\
\cline { 2 - 5 } & & $\begin{array}{l}\text { Treated or } \\
\text { screened (\%) }\end{array}$ & $\begin{array}{l}\text { No } \\
\text { intervention (\%) }\end{array}$ \\
\hline Age, years & $67-75$ & 9.11 & 38.06 & All (\%) \\
& $76-85$ & 9.02 & 28.47 & 47.17 \\
& $>86$ & 2.87 & 12.47 & 37.49 \\
Sex** & Female & 17.06 & 50.21 & 15.34 \\
& Male & 3.94 & 28.79 & 67.27 \\
Diabetes & No & 17.23 & 63.41 & 32.73 \\
& Yes & 3.77 & 15.59 & 80.64 \\
Cancer & No & 17.56 & 68.66 & 19.36 \\
& Yes & 3.45 & 10.34 & 86.22 \\
CKD & No & 20.75 & 76.70 & 13.78 \\
& Yes & 0.25 & 2.30 & 97.45 \\
& & & & 2.55 \\
\hline
\end{tabular}

Note: $* * p<0.01$.

Abbreviation: CKD, chronic kidney disease.

In the included patients, 1,007 fractures were sustained. The most common fractures encountered were closed vertebral fractures (ICD-9 codes 805.2 and 805.4) and closed femoral neck fractures (ICD-9 code 820.8). A total of 267 patients were diagnosed with various osteopathies, including nonunion or stress fractures. A total of 469 patients underwent orthopedic procedures to correct the fractures mentioned previously (Figure 1).
As expected, fractures were seen more frequently in older female patients than in their male counterparts. This is similar to previously published data. ${ }^{7}$ Of the 1,219 patients, 820 $(67.2 \%)$ were females and 399 (32.7\%) were males. This was an expected sex difference, which has been documented in prior studies. ${ }^{4,8}$ The ages in which males and females sustained their fractures were similar. The mean age for females was 77.76 years in comparison to 75.78 in males (Table 2).

Of the 1,219 patients studied, treatment or screening for osteoporosis by BMD occurred in 256 (21.0\%) patients. Treatment medications (bisphosphonate therapy, teriparatide, calcitonin, raloxifene, and estrogen replacement) were received by $219(18.0 \%)$ patients and immunologic agent, denosumab, by $31(2.5 \%)$ patients. Alendronate was the most common treatment received by 92 patients (Figure 2). When looking at osteoporosis screening and treatment after a fracture, males underwent less osteoporosis testing than females. Of the 399 males studied, only 48 (12.0\%) either received $\mathrm{BMD}$ or treatment for osteoporosis following a fracture. Conversely, 208/820 (25.4\%) females during the same time period received BMD evaluation or osteoporosis treatment; this was statistically significant with $P<0.05$ (Figure 3). There was no association between incidence of

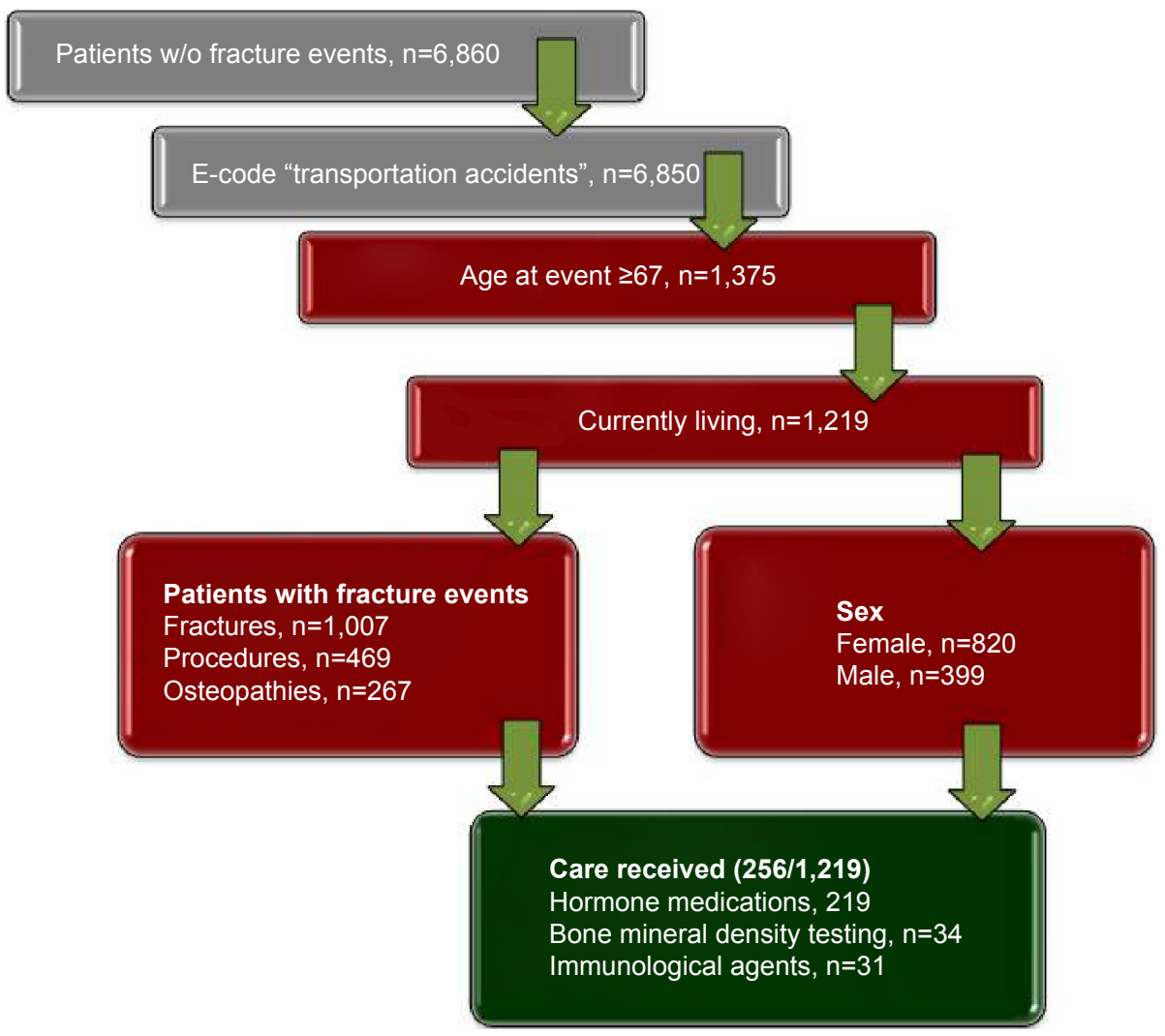

Figure I PHM2 - osteoporosis management in older patients, experiencing a fracture from February 02, 2013 to July $31,2014$. Abbreviations: $n$, number of patients; w/o, without. 
Table 2 PHM2 - osteoporosis management in older patients, experiencing a fracture from June 0I, 2013 to November II, 20 I4

\begin{tabular}{|c|c|c|c|c|c|c|c|c|}
\hline & \multirow[t]{2}{*}{ Age at onset } & \multicolumn{2}{|l|}{ Sex } & \multicolumn{5}{|l|}{ Race } \\
\hline & & Female & Male & Am Indian & Asian & Black & Other & White \\
\hline Min & 67 & 67 & 67 & 67 & 68 & 67 & 67 & 67 \\
\hline First quartile & 71 & 71 & 70 & 67.5 & 71 & 71 & 70.5 & 71 \\
\hline Median & 76 & 77 & 75 & 68 & 73 & 76.5 & 78 & 76 \\
\hline Mean & 77.11 & 77.76 & 75.78 & 70 & 76.8 & 77.6 & 79.03 & 76.99 \\
\hline Third quartile & 83 & 83 & 81 & 71.5 & 83 & 84 & 87 & 82 \\
\hline $\operatorname{Max}$ & 102 & 102 & 96 & 75 & 95 & 102 & 97 & 101 \\
\hline Count & 1,219 & 820 & 399 & 3 & 15 & 86 & 59 & 1,056 \\
\hline
\end{tabular}

Abbreviations: Min, minimum; Max, maximum.

being screened or treated with the comorbid conditions of diabetes, malignancy, and chronic renal disease. In addition, age of the individual did not influence the treatment plan (Table 1).

Data were also obtained regarding the time of the year the index fracture was sustained. The least number of fractures occurred in February 2014; however, the largest number of fractures were seen in December 2013. Overall, fractures appeared to occur throughout the entire calendar year at a steady rate.

\section{Discussion}

Our research extracted data from our electronic medical record system and billing databases, and analyzed the rate of subsequent bone mineral testing or treatment for osteoporosis in patients sustaining low trauma fractures. Testing or treatment occurred for $25.4 \%$ of females, $12.0 \%$ of males, and $21.0 \%$ for all subjects. This is consistent with the national average of osteoporotic fracture management in females, as published in the 2012 Medicare report provided by Centers for Medicare and Medicaid Services. ${ }^{9}$

When we compared our study to previous large insurance claims data published recently, results are very similar. In addition to medications used, we were able to capture the rate of bone densities done in this high-risk population 18 months after the initial fracture. The data consistently shows an unmet need for the appropriate management of post-osteoporotic fracture. ${ }^{10}$

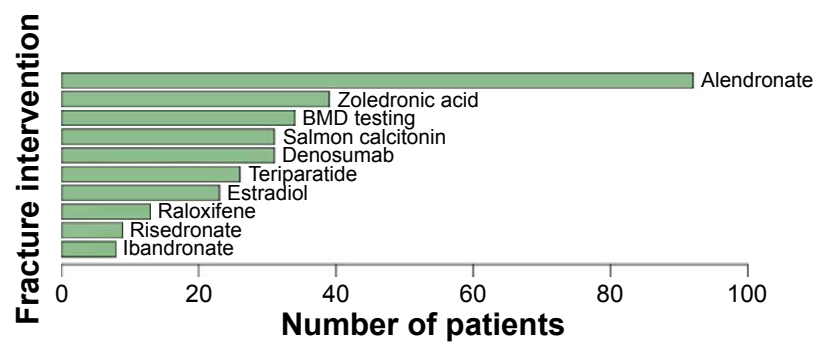

Figure 2 PHM2 - osteoporosis management in older patients, experiencing a fracture (top ten treatments) from June 0I, 2013 to November II, 2014.

Abbreviation: BMD, bone mineral density.
There has been increased emphasis on quality measures in health care in the USA in recent years, including evaluation and treatment of osteoporosis. ${ }^{11,12}$ This was recently reflected in a letter to the New England Journal of Medicine by Ms Burwell, US Secretary of Health and Human Services. ${ }^{13}$ She stated that Medicare has goal of linking fee-for-service payments to quality or value of $85 \%$ by 2016 and $90 \%$ by 2018. In the Medicare population, post-fracture evaluation and treatment rates for females are often $<25 \%$, based on data provided by National Committee for Quality Assurance. ${ }^{9}$ With data showing that by 2025 , annual fracture costs are expected to rise by almost $50 \%$ and incur US $\$ 25.3$ billion in health care costs, it is becoming imperative to ensure these patients are receiving proper evaluation and treatment for osteoporosis. ${ }^{5}$

As the use of electronic medical records is becoming more widespread throughout the USA, it is more feasible to identify patients quickly with fractures to ensure quality health care. It has been demonstrated that inpatient Medicare costs could be reduced by US $\$ 115.41$ million and long-term care costs by US $\$ 43.51$ million in 3 years if an additional 1 million females would be simply screened for osteoporosis, ultimately preventing 35,000 fractures. ${ }^{10}$

Our university hospital data reflect the results of the national data, in which the majority of these fractures are evaluated by orthopedic surgeons. Oftentimes, following the presentation of fracture, medical management for osteoporosis is not started. There is a lack of coordination of care. The American Society of Bone Mineral Research published

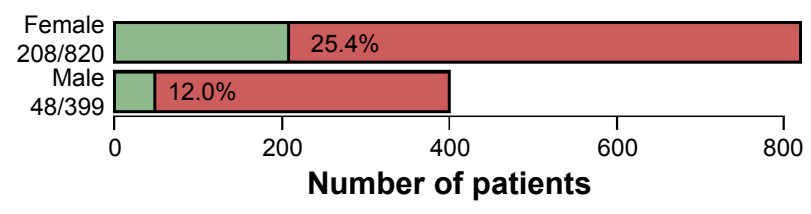

Figure 3 PHM2 - osteoporosis management in older patients, experiencing a fracture from June 01,2013 to November II, 2014.

Note: Living female and male at least 67 years old, who suffered a fracture within the 18 months (shown in red and green), and have received bone mineral density testing or osteoporosis medication afterward (shown in green). 
a report discussing the importance of a fracture liaison service to engage high-risk patient with pathways to treatment. A multidisciplinary approach is needed to achieve quality care in post-fracture management. Previous studies have shown that fracture liaison service has increased the rate of treatment post-fracture to $80 \%{ }^{11}$

There are several limitations to consider. This is retrospective data extraction that may have inaccuracies based on coding and misdiagnosis. Also, we were not able to follow these patients after discharge from the hospital to outside institutions. They may have had BMD or treatment at another medical office or medical center unrelated to our institution. Thus, our data would underestimate the total number of individuals who received the primary outcome. We were unable to exclude the possibility that, the severity of illness could preclude individuals from appropriate follow-up and therefore limit access to care.

The strength of our study included the 1,219 patients who sustained a fracture. We were able to collect basic information on demography and some comorbidities. In addition, the electronic medical record allowed us to identify that osteoporosis medications were prescribed for our cohort. The uniqueness to our study compared to prior studies includes the comparison of males in addition to females as occurring in most traditional osteoporotic studies. ${ }^{9}$

\section{Conclusion}

There is a great need to create clinical pathways to coordinate effective and efficient care. This in turn will help patient outcomes and reduce health care costs. Further research is needed to determine the optimal mechanism to identify these patients to ensure proper screening and treatment of osteoporosis.

\section{Acknowledgments}

This work was supported in part by a Clinical and Translational Science Award grant from National Center for Research Resources (NCRR) and National Center for Advancing Translational Sciences (NCATS) awarded to the University of Kansas Medical Center for Frontiers: The Heartland Institute for Clinical and Translational Research \# UL1RR033179, which is now at NCATS \# UL1TR000001.

The contents are solely the responsibility of the authors and do not necessarily represent the official views of the National Institutes of Health or NCATS.

Expressing observations from electronic medical record flowsheets in an $\mathrm{i} 2 \mathrm{~b} 2$ based clinical data repository to support research and quality improvement. ${ }^{14}$

\section{Author contributions}

All authors contributed toward data analysis, drafting and revising the paper and agree to be accountable for all aspects of the work.

\section{Disclosure}

Rajib K Bhattacharya is a promotional speaker for Abbvie and Shire. The authors report no other conflicts of interest in this work.

\section{References}

1. Elliot-Gibson V, Bogoch ER, Jamal SA, Beaton DE. Practice patterns in the diagnosis and treatment of osteoporosis after a fragility fracture: a systematic review. Osteoporosis Int. 2004;15(10):767-778.

2. Liu SK, Munson JC, Bell JE, et al. Quality of osteoporosis care of older Medicare recipients with fragility fractures: 2006 to 2010.J Am Geriatr Soc. 2013;61(11):1855-1862.

3. Mehrpour SR, Aghamirsalim MR, Sorbi R. Are hospitalized patients with fragile fractures managed properly in relation to underlying osteoporosis? J Clin Rheumatol. 2012;18(3):122-124.

4. Antonelli M, Einstadter D, Magrey M. Screening and treatment of osteoporosis after hip fracture: comparison of sex and race. $J$ Clin Densitom. 2014;17(4):479-483.

5. Burge R, Dawson-Hughes B, Solomon DH, Wong JB, King A, Tosteson A. Incidence and economic burden of osteoporosis-related fractures in the United States, 2005-2025. J Bone Miner Res. 2007;22(3): 465-475.

6. Bone Health and Osteoporosis: A Report of the Surgeon General Reports of the Surgeon General. Rockville, MD, USA; 2004.

7. Looker AC, Borrud LG, Dawson-Hughes B, Shepherd JA, Wright NC Osteoporosis or low bone mass at the femur neck or lumbar spine in older adults: United States, 2005-2008. NCHS Data Brief. 2012;(93):1-8.

8. Blecher R, Wasrbrout Z, Arama Y, Kardosh R, Agar G, Mirovsky Y. Who is at risk of receiving inadequate care for osteoporosis following fragility fractures? A retrospective study. Isr Med Assoc J. 2013;15(10): 634-638.

9. Assurance NCfQ. The State of Health Care Quality 2010. Available from: http://www.ncqa.org/report-cards/health-plans/state-of-healthcare-quality. Accessed August 1, 2016.

10. Wilk A, Sajjan S, Modi A, Fan CP, Mavros P. Post-fracture pharmacotherapy for women with osteoporotic fracture: analysis of a managed care population in the USA. Osteoporosis Int. 2014;25(12):2777-2786.

11. King AB, Saag KG, Burge RT, Pisu M, Goel N. Fracture reduction affects medicare economics (FRAME): impact of increased osteoporosis diagnosis and treatment. Osteoporosis Int. 2005;16(12):1545-1557.

12. Solomon DH, Patrick AR, Schousboe J, Losina E. The potential economic benefits of improved postfracture care: a cost-effectiveness analysis of a fracture liaison service in the US health-care system. J Bone Miner Res. 2014;29(7):1667-1674.

13. Burwell SM. Setting value-based payment goals - HHS efforts to improve U.S. health care. N Engl J Med. 2015;372(10):897-899.

14. Waitman LR, Warren JJ, Manos EL, Connolly DW. Expressing observations from electronic medical record flowsheets in an i2b2 based clinical data repository to support research and quality improvement. AMIA Annu Symp Proc. 2011;2011:1454-1463. Epub 2011 Oct 22. 


\section{Publish your work in this journal}

Clinical Interventions in Aging is an international, peer-reviewed journal focusing on evidence-based reports on the value or lack thereof of treatments intended to prevent or delay the onset of maladaptive correlates of aging in human beings. This journal is indexed on PubMed Central, MedLine,

CAS, Scopus and the Elsevier Bibliographic databases. The manuscript management system is completely online and includes a very quick and fair peer-review system, which is all easy to use. Visit http://www.dovepress. com/testimonials.php to read real quotes from published authors. 